\title{
Regenerative Blower for EVA Suit Ventilation Fan
}

\author{
Michael G. Izenson ${ }^{1}$ and Weibo Chen ${ }^{2}$ \\ Creare Incorporated, Hanover, $\mathrm{NH}, 03755$ \\ and \\ Heather L. Paul ${ }^{3}$ \\ NASA Lyndon B. Johnson Space Center, Houston, TX, 77058
}

\begin{abstract}
Portable life support systems in future space suits will include a ventilation subsystem driven by a dedicated fan. This ventilation fan must meet challenging requirements for pressure rise, flow rate, efficiency, size, safety, and reliability. This paper describes research and development that showed the feasibility of a regenerative blower that is uniquely suited to meet these requirements. We proved feasibility through component tests, blower tests, and design analysis. Based on the requirements for the Constellation Space Suit Element (CSSE) Portable Life Support System (PLSS) ventilation fan, we designed the critical elements of the blower. We measured the effects of key design parameters on blower performance using separate effects tests, and used the results of these tests to design a regenerative blower that will meet the ventilation fan requirements. We assembled a proofof-concept blower and measured its performance at sub-atmospheric pressures that simulate a PLSS ventilation loop environment. Head/flow performance and maximum efficiency point data were used to specify the design and operating conditions for the ventilation fan. We identified materials for the blower that will enhance safety for operation in a lunar environment, and produced a solid model that illustrates the final design. The proof-ofconcept blower produced the flow rate and pressure rise needed for the CSSE ventilation subsystem while running at $5400 \mathrm{rpm}$, consuming only $9 \mathrm{~W}$ of electric power using a non-optimized, commercial motor and controller and inefficient bearings. Scaling the test results to a complete design shows that a lightweight, compact, reliable, and low power regenerative blower can meet the performance requirements for future space suit life support systems.
\end{abstract}

$\begin{array}{ll}\mathrm{N} & \text { Impeller rotating speed }\left(\mathrm{s}^{-1}\right) \\ \mathrm{N}_{\mathrm{s}} & \text { Specific speed }(-) \\ \mathrm{Q} & \text { Volumetric flow rate }\left(\mathrm{m}^{3} / \mathrm{s}\right) \\ \Delta \mathrm{p} & \text { Pressure rise }(\mathrm{Pa}) \\ \rho & \text { Gas density }\left(\mathrm{kg} / \mathrm{m}^{3}\right) \\ \mathrm{d}_{\mathrm{o}} & \text { Impeller diameter }(\mathrm{m})\end{array}$

Nomenclature

U Impeller tip speed $(\mathrm{m} / \mathrm{s})$

$\mathrm{A}_{\mathrm{c}} \quad$ Channel flow area $\left(\mathrm{m}^{2}\right)$

$\psi \quad$ Head coefficient (-)

$\varphi \quad$ Flow coefficient (-)

\section{Introduction}

The space suit system needs a fan to force ventilation gas through the pressure garment and the Portable Life Support System (PLSS). The current design for the Constellation Space Suit System (CSSS) includes a dedicated ventilation fan, which is a departure from prior space suit designs. As a result, developing the ventilation fan is a critical technology need. The ventilation fan must produce a pressure rise on the order of $1 \mathrm{kPa}$ to force the required ventilation flow through the pressure garment and PLSS, and conventional centrifugal or axial blowers cannot

\footnotetext{
${ }^{1}$ Principal Engineer, P.O. Box 71, Senior Member of AIAA.

${ }^{2}$ Engineer, P.O. Box 71.

${ }^{3}$ Constellation Space Suit PLSS Ventilation Subsystem Lead, Space Suit and Crew Survival Systems Branch, Crew and Thermal Systems Division, 2101 NASA Parkway, Mail code EC5.
} 
achieve this pressure rise at low speed and with high reliability. A regenerative blower, which provides multiple stages of pressurization using a single, low-speed impeller, is uniquely suited for exploration space suits.

Constellation Space Suit System. Although extravehicular activity (EVA) suits have been used extensively in the Apollo, Space Shuttle, and International Space Station programs, these prior space suits are not suitable for future space exploration missions. The National Aeronautics and Space Administration (NASA) needs new space suit technology to meet all exploration mission requirements, improve reliability, reduce logistical and maintenance burdens, and reduce life cycle costs. As a result, the Constellation Program has adopted a design philosophy for EVA suits that calls for: (1) a single space suit system with two configurations (launch/entry/abort/microgravity and lunar surface EVA) that share many components, (2) a common evolvable infrastructure, (3) a minimum set of hardware that meets all mission phase requirements, and (4) a modular, reconfigurable, component-based open architecture. ${ }^{1}$ NASA has completed studies to define the optimum design for the exploration EVA suit's portable life support system. ${ }^{2}$

Ventilation Flow Requirements. Unlike prior space suit systems, future exploration systems will include a ventilation subsystem with its own fan (Fig. 1). ${ }^{3}$ The fan must draw the required ventilation flow from the pressure garment via the liquid cooling and ventilation garment (LCVG) and force it through the $\mathrm{CO}_{2}$ and humidity control system, a humidifying heat exchanger, in-line filters, and then back into the helmet. The required ventilation flow rate is selected to ensure adequate washout of $\mathrm{CO}_{2}$ from the helmet. The ventilation fan must produce this flow rate while generating enough pressure rise to overcome the combined flow resistance of all the components in the ventilation subsystem. Taking these requirements into account and adding appropriate margins, NASA has derived baseline design requirements ${ }^{4}$ for the ventilation fan. Table 1 lists the key requirements. In addition to challenging performance and size requirements, extremely high reliability is critical for space exploration. The service interval is specified to allow for one hundred EVAs of eight hours each with a safety factor of three, yielding the 2500 hour requirement.

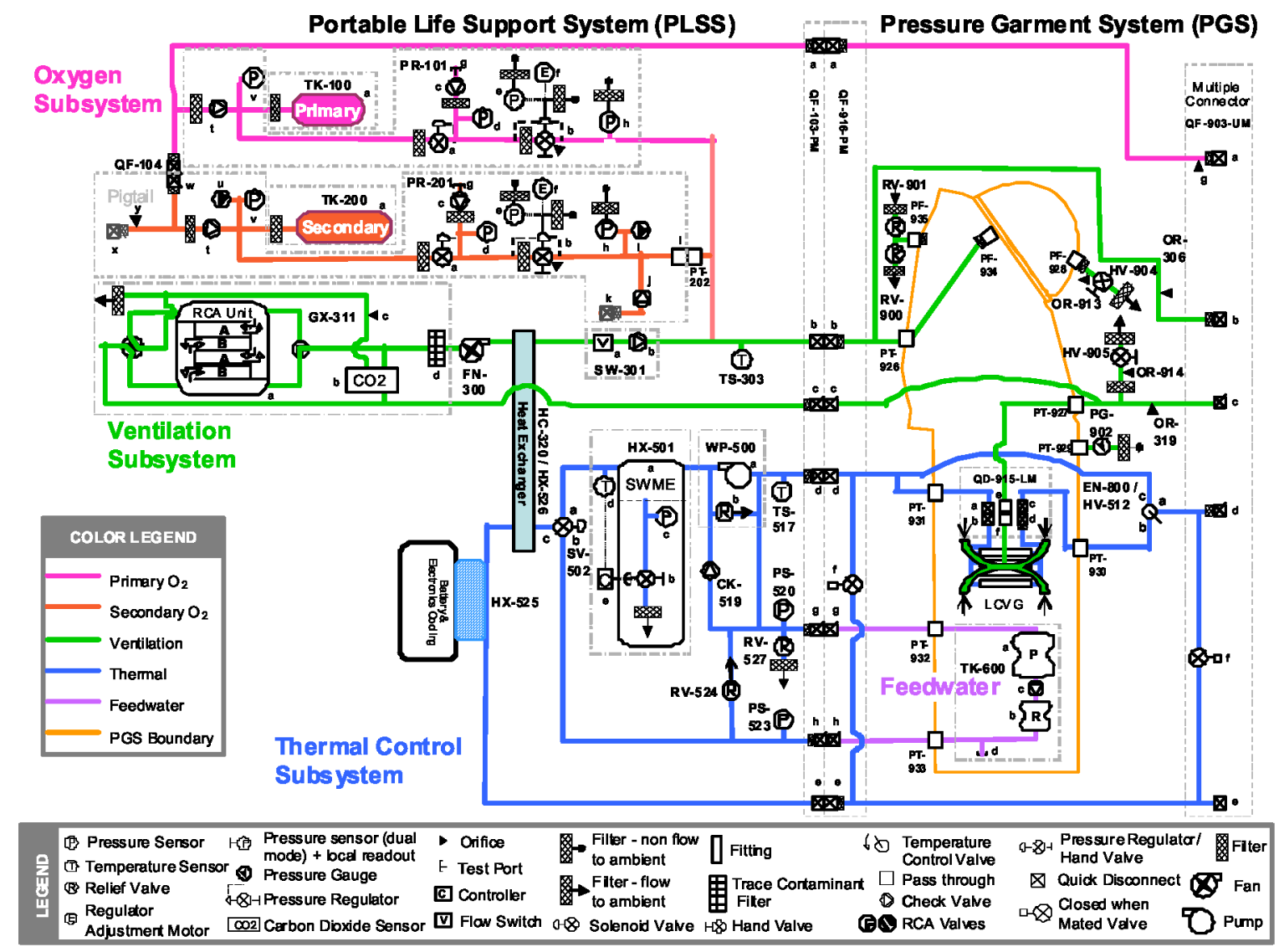

Figure 1. Baseline PLSS Schematic for Constellation EVA Suit ${ }^{3}$ 
Table 1 lists current design requirements for the baseline Constellation EVA suit, as well as a projected high flow rate case and a requirement for "buddy mode" operation. In buddy mode, a single ventilation fan circulates oxygen through two EVA suits in the event that one of the suit's fan has failed. The flow rate in buddy mode is twice the baseline level and the pressure rise has increased by a factor of 2.5 to account for additional losses from coupling two EVA suits together. The maximum power requirement is relaxed in this mode, so that the fan can operate less efficiently while providing the increased ventilation flow.

\begin{tabular}{|c|c|c|c|}
\hline Helmet Flow Rate Requirement & $\begin{array}{c}\text { Current } \\
\text { Baseline }\end{array}$ & $\begin{array}{c}\text { High Flow } \\
\text { Rate }\end{array}$ & Buddy Mode \\
\hline Operating pressure (psia) & 4.3 & 4.3 & 4.3 \\
\hline Volumetric flow rate (actual $\mathrm{ft}^{3} / \mathrm{min}$ ) & 4.7 & 5.9 & 9.4 \\
\hline$\Delta \mathrm{P}$ in space suit (in. $\mathrm{H}_{2} \mathrm{O}$ ) & 2.7 & 4.1 & 6.75 \\
\hline$\Delta \mathrm{P}$ at $1 \mathrm{~atm}$ pressure (in. $\mathrm{H}_{2} \mathrm{O}$ ) & 9.2 & 14.0 & 23.1 \\
\hline Maximum power consumption (W) & 8.0 & - & - \\
\hline Aero power at suit pressure $(\mathrm{W})$ & 1.5 & 2.8 & 7.4 \\
\hline Maximum mass (w/out controller) $(\mathrm{kg})$ & \multicolumn{3}{|c|}{$0.91^{4}$} \\
\hline Maximum size (L) & \multicolumn{3}{|c|}{0.49} \\
\hline Service life (hr) & \multicolumn{3}{|c|}{2500} \\
\hline
\end{tabular}

Oxygen Environment. The ventilation fan operates in a pure oxygen environment in the PLSS. As a result, special care is needed to ensure that the materials are compatible with oxygen and that operation of the blower cannot result in an oxygen fire. Oxygen-compatible plastics (such as poly(amide-imides)) are preferred for the impeller, since lunar dust particles may impact a rotating metal impeller and cause sparks, although care must be taken to avoid potential static buildup. A maximum impact speed of $30 \mathrm{~m} / \mathrm{s}$ has been identified as a safe operating limit. ${ }^{5}$ The bearings and lubricant must be compatible for extended exposure to an oxygen environment.

Conventional Blower Technology Is Not Adequate. Kempf et al. surveyed a wide variety of available blowers and fans to identify candidates for the CSSS PLSS ventilation subsystem. They found that conventional air blowers can meet the ventilation flow requirements only by operating at high rotating speed. A conventional centrifugal blower can achieve the required pressure rise only by operating with a very high tip speed $(\sim 60 \mathrm{~m} / \mathrm{s})$. However, for efficient operation at the flow rate range targeted for the ventilation fan, the diameter of the impeller also needs to be relatively small $(<12.5 \mathrm{~cm})$. Consequently, a centrifugal blower needs to operate at a very high rotational speed to meet the performance requirements. As a result, the leading candidate, a commercial-off-the-shelf centrifugal blower (Micronel U51D1-024KK-5), needs to rotate at a speed of approximately 43,000 rpm to produce the pressure rise needed to push the required flow through the ventilation loop. Likewise, the gas-bearing axial fan would need to spin at approximately $166,000 \mathrm{rpm}$ to generate enough pressure rise. These high tip speeds can ignite fires due to particle impact in an oxygen environment. High-speed operation also reduces lifetime and tends to be very noisy. The high-speed axial fan would be difficult to repair and maintain without elaborate tools. It would be very difficult to further increase the operating speed of these existing blowers to safely meet the flow requirements for buddy mode operation.

Context. To investigate innovative alternatives to conventional fans, NASA Johnson Space Center (JSC) awarded a Phase I Small Business Innovation Research contract to Creare Inc. in 2009 to show the feasibility of meeting the CSSS ventilation fan requirements using a regenerative blower. This paper summarizes the results of that study, which included an investigation of fabrication methods, separate effects tests of blower components, blower design optimization, assembly and testing a proof-of-concept blower, and design of a Phase II prototype. The Phase II program, which began in January 2010, aims to build a prototype ventilation fan that meets CSSS requirements, demonstrate performance and reliability in simulated space suit environments, and deliver the prototype to NASA for further evaluation in the PLSS ventilation laboratory.

\section{A. Analysis of Ventilation Blower Performance Requirements}

A regenerative blower can meet ventilation flow requirements while running efficiently and reliably at low speed. A survey of all blower designs shows that the regenerative blower should have the highest efficiency for this application. A regenerative blower achieves higher pressure rises than centrifugal or axial blowers because the air is

${ }^{4}$ CRAVE DO-39 PLSS Fan Assembly Development for Exploration Technology, Contract no. NNJ05HB39B, Fan Assembly Concept and Design Report, Hamilton Sundstrand, Final Report. 
energized by the impeller more than once as it flows through a regenerative machine. Prior tests of similar-sized blowers confirm this capability.

A Regenerative Blower Can Achieve High Efficiency at Low Speed. Any fan or blower can achieve a wide range of head/flow operating points if it rotates at the appropriate speed. However, there is no guarantee of efficient operation, since a fan or blower achieves maximum efficiency at a given flow rate over only a limited range of operating speeds. Figure 2 illustrates this general characteristic of turbomachines using hypothetical performance characteristics. At a fixed speed, a fan or blower will have a fixed relationship between flow rate and pressure rise (solid lines). This head/flow characteristic will vary depending on the rotating speed of the impeller. In general the flow rate is directly proportional to the rotating speed and the pressure rise varies with the square of the rotating speed. However, turbomachines operate efficiently only when losses are low and the internal flows align well with the geometry of the rotating blades and stationary flow passages. The dashed lines in Fig. 2 show schematically that the most efficient operating point for a given turbomachine will correspond to a particular rotating speed at a particular flow rate and pressure rise.

If a blower's performance is characterized not only by pressure rise and flow rate but also by best efficiency, then the performance map in Fig. 2 collapses to a single point that can be expressed in terms of dimensionless parameters. Two dimensionless parameters can be computed that summarize the performance at best efficiency: the specific speed $\left(N_{s}\right)$ and the specific diameter $\left(D_{s}\right)$ :

$$
N_{s}=\frac{2 \pi N Q^{0.5}}{(\Delta p / \rho)^{0.75}} \quad D_{s}=\frac{d_{0}(\Delta p / \rho)^{0.25}}{Q^{0.5}}
$$

These dimensionless quantities characterize the performance of particular classes of turbomachines. They can be computed from head/flow and size/speed requirements, then used to identify the type of blower that can achieve that head/flow requirement at best efficiency. For example, Fig. 3 shows efficiency data (at best efficiency) for a range of turbomachinery types plotted as a function of specific speed and specific diameter. Using the head/flow conditions listed in Table 1, and assuming reasonable ranges for head coefficient and speed, we have plotted representative points showing design of centrifugal and regenerative blowers along with characteristic curves that show efficiencies for regenerative and centrifugal blowers. The aerodynamic efficiencies of these two types of blowers are comparable, both in the range of 50-60\%. However, since the head coefficient of a regenerative blower is about ten times larger than that of a radial blower, a regenerative blower can operate at a much slower rotating speed. Lower speed will substantially increase reliability, simplify construction, and reduce noise. Since the data in Fig. 3 come from large turbomachines, the actual aerodynamic efficiency that can be achieved by a small ventilation blower will be lower due to increased losses. However the general efficiency trends should still be valid at a smaller scale.

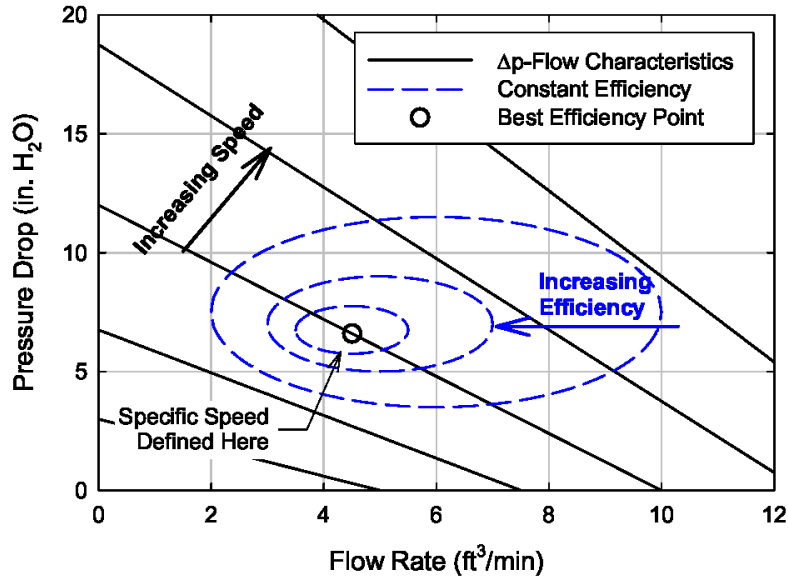

Figure 2. General Performance Characteristics of Turbomachines.

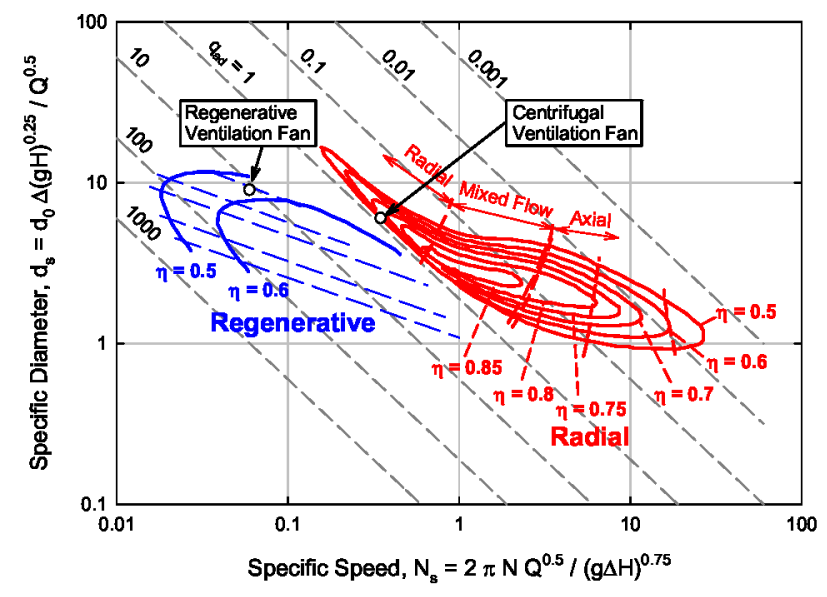

Figure 3. Comparison of Regenerative and Centrifugal Blowers for EVA Suit Ventilation. 


\section{B. Regenerative Blower Concept}

The regenerative blower is attractive mainly because the ventilation fan must generate a relatively large pressure rise to force the ventilating gas through several process steps in the PLSS. Figure 4 illustrates the main components in a regenerative blower and illustrates why a regenerative blower is an attractive choice. A regenerative blower boosts the pressure of the gas repeatedly because it flows through the blades multiple times, enabling relatively high pressure rise for a given flow rate. The figure shows the simplest embodiment, in which the blower impeller is a flat disk with a single row of blades oriented radially on its rim. Gas flows from the inlet to the outlet through a toroidal flow channel that includes the blades. The rotating impeller blades transfer momentum to the gas and impel it on a spiral path through the toroidal flow channel. The gas passes through the rotating blades several times before leaving the blower, each time receiving an additional boost in total energy. In this way the blower provides multiple stages of pressure rise in a single device. This is the reason that a regenerative blower can produce a higher pressure rise than a centrifugal blower with the same diameter running at the same rotating speed.

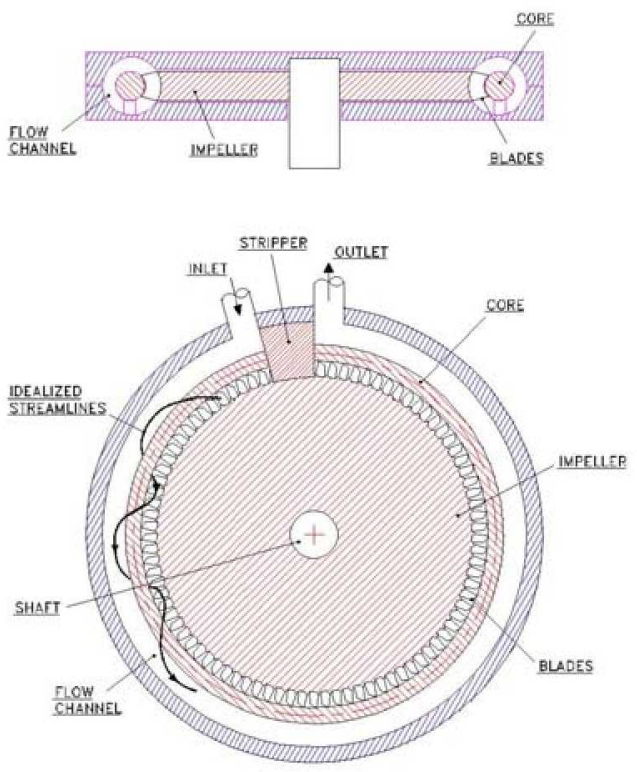

(a) Schematic

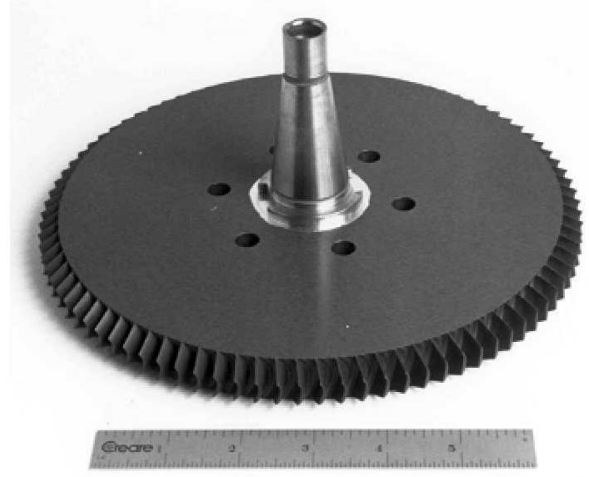

(b) Impeller From a Regenerative Compressor

\section{Figure 4. Regenerative Blower Concept.}

Operating Principles. The regenerative blower imparts momentum to a gas stream by changing the gas velocity as it flows through the row of blades. Gas entering the blades has a relatively low tangential velocity, while the gas leaving the blades has a higher tangential velocity due to the shape of the blades. This change in angular momentum requires work input from the blade row. As the exit gas flows through the toroidal flow channel to reenter the blades, the angular velocity decreases due to diffusion. That is, as the gas flows through the toroidal chamber its velocity decreases and static pressure increases. By the time the gas reaches the inlets of the blades again, its tangential velocity has decreased to the same level it had before entering the blades the last time, while its static pressure has increased. First-order design methods based on this type of analysis have been published by Sixsmith, ${ }^{7}$ Swift, ${ }^{8}$ and several others.

Early Demonstrations. Prior to the ventilation fan application, we had already demonstrated that a small regenerative blower can achieve the CSSS ventilation flow requirements at relatively low speed. Figure $5 \mathrm{a}$ is a photograph of a small regenerative blower built to demonstrate the technology for a similar application, and Fig. 5b shows the normalized head/flow performance at rotating speeds ranging from 1770 to $7340 \mathrm{rpm}$. This non-optimized blower essentially achieved the baseline ventilation requirements at a rotating speed of approximately $5000 \mathrm{rpm}$ and can achieve the future requirements at approximately $7000 \mathrm{rpm}$. At rotating speeds from 4000 to $6000 \mathrm{rpm}$, the blower achieved mechanical efficiencies greater than $30 \%$ and total efficiencies (including an off-the-shelf motor and inverter) in the range $20-23 \%$. 


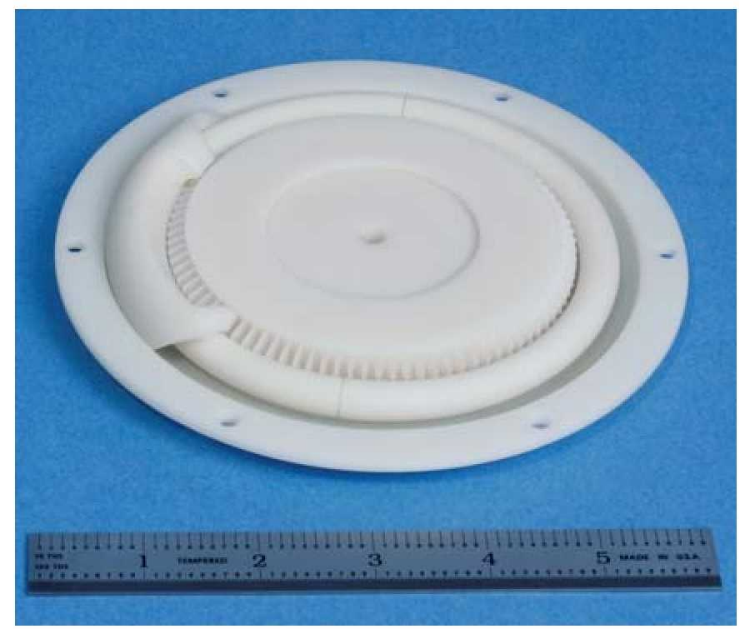

(a) Impeller and Blower Housing (top cover of housing is removed)

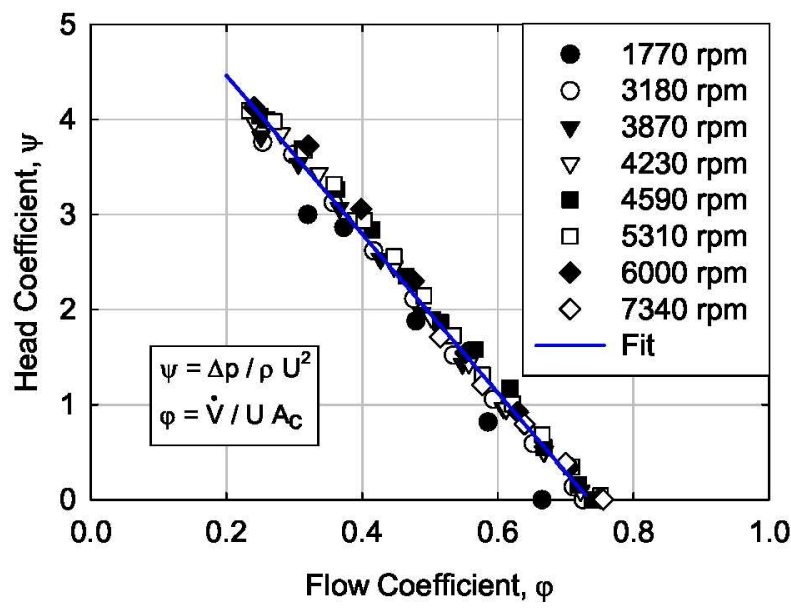

(b) Head/Flow Performance Data

Figure 5. Early Model Regenerative Blower.

Design Methods. We have developed design methods to analyze the performance of regenerative blowers. During operation, the impeller blades transfer momentum to the gas that flows through the blower. Blade inefficiency, flow friction, and leakage prevent the blower from reaching its ideal performance. The design models account for the following phenomena:

- Actual (as opposed to ideal) momentum transfer from the blades to the gas.

- Form losses at the inlet and exit of the blower.

- Flow friction in the toroidal flow path and between the blades.

- Impeller drag.

- Leakage from the exit side of the impeller to the inlet side.

- Gas carryover through the stripper seal.

- Motor inefficiency.

The ideal performance of the blower depends on the transfer of momentum from the blades to the gas. We use both computational fluid dynamics (CFD) (Fig 6a) and two-dimensional boundary layer/potential flow analysis (Fig. 6b) to calculate the flow through the blades. Both of these analysis approaches have attractive features along with limitations. CFD calculations yield a complete, two- or three-dimensional field of velocity and pressure through the blades, and can be used to calculate both the average change in gas velocity and losses due to friction through the blades. However, CFD results are difficult to verify experimentally and rely on assumption made about gridding, turbulence, and boundary conditions. The boundary layer/potential flow models use commercial software that is based on many years of experience designing axial-flow turbomachines. These models yield surface velocity distributions, net momentum change, and calculated parameters that indicate how close the flow might be to separation. However these models are based mainly on data from much larger machines, and scaling to the size of the ventilation fan is uncertain. Therefore we use both the CFD and potential flow analysis in conjunction with test data to assess blade geometry and the flow through the blades.

We calculate losses using simple, one-dimensional models for the relevant flow phenomena that employ empirical constants to account for the specific blower geometry. Form losses at the inlet and exit to the blower are proportional to the local dynamic head and are calculated using tabulated loss coefficients for similar configurations. Frictional losses due to flow in the toroidal channel and between the blades are estimated using friction factors based on standard relations and the local Reynolds number. Impeller drag is mainly due to viscous drag between the impeller and housing faces and is computed using standard relations for viscous losses between rotating disks. Losses arise due to gas carryover through the stripper seal because each blade pair will carry a small amount of gas through the stripper from the high-pressure to the low-pressure section of the flow channel. We estimate motor efficiency using first order calculations of resistive losses in the stator windings based on the coil design and the number of amp-turns required to produce the magnetic flux to drive the impeller. 


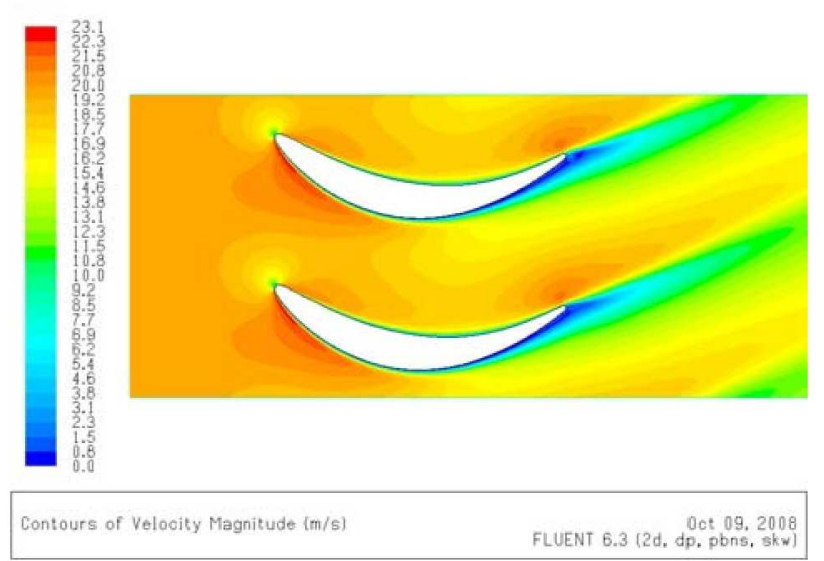

(a) Computational Fluid Dynamics

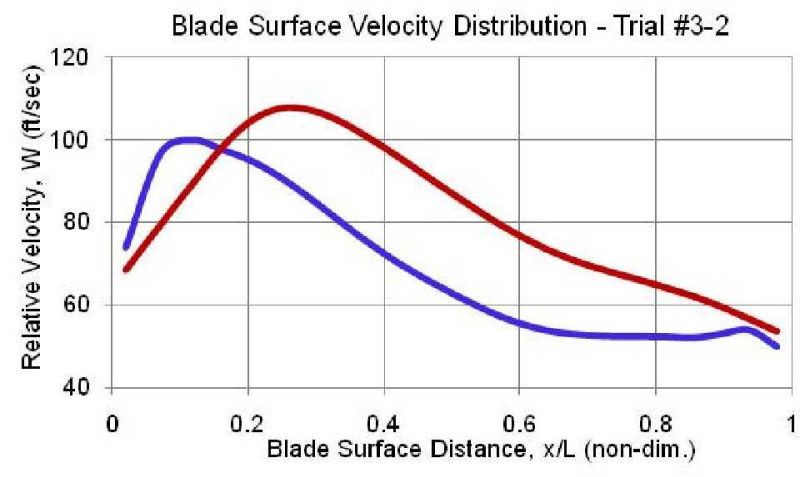

(b) 2-D Boundary Layer/Potential Flow Analysis

Figure 6. Blade Analysis Methods.

\section{Design Objectives}

The objectives of the blower development effort are to produce a compact and lightweight ventilation fan that runs at low speed with high reliability and high efficiency.

- Compact and Lightweight. The goal is to produce a ventilation fan with a diameter less than $13.5 \mathrm{~cm}$ ( $5.3 \mathrm{in}$.), overall volume less than $0.36 \mathrm{~L}\left(22 \mathrm{in}^{3}{ }^{3}\right)$, and a mass less than $0.4 \mathrm{~kg}(0.9 \mathrm{lb})$. These numbers are based on our performance prediction, and meet or exceed the requirements in Table 1.

- Low Speed. We aim to produce a regenerative blower that meets requirements for the ventilation subsystem while rotating at less than $5400 \mathrm{rpm}$.

- Safe. The blower should be built from oxygen-compatible materials that will not generate sparks to prevent the possibility of oxygen fire.

- High Reliability. Low rotating speed will enable very high reliability for the required service life of $2500 \mathrm{hrs}$. Materials must be chosen that will not degrade in an oxygen environment.

- High Efficiency. The goal for the blower is to meet the head/flow requirements for the ventilation subsystem while consuming less than $8 \mathrm{~W}$ during normal operation in a space suit environment.

- Low Noise. Noise generated in turbomachines depends strongly on the rotating speed. By minimizing the rotational speed of the regenerative blower, noise will also be minimized.

- Easy to Maintain. The regenerative blower will include no high-precision or specialized components. It will be easy to inspect, repair, maintain, and reassemble in remote locations.

\section{Development and Testing}

Early development of the regenerative blower involved separate effects tests, integral blower tests, and component design.

Separate Effects Tests. We used a test bed for regenerative blower components to measure the effects of blade geometry, blade spacing, and flow passage design on performance of the blower. We used data from these tests to specify the design of the prototype blower.

Figure 7 and Fig. 8 illustrate the test bed for components of regenerative blowers. Figure 7 shows the test bed blower, which includes two parallel sets of impeller blades. Components for the test bed are generally fabricated by stereolithography, although metal parts can be tested as well. The schematic in Fig. 8 shows the test setup. We provide a metered flow of air from a shop compressor and measure the pressure rise produced by the blower. This test arrangement enables measurement of the flow rate upstream from the blower, so that we can test the complete range of blower flows despite a potentially large pressure drop across the flow meter (there is no other way to measure the flow rate at zero pressure rise). The motor is mounted on bearings and the reaction torque is measured from the force exerted by the torque arm on an electronic balance. We measure rotating speed with a strobe light. With this setup, we can measure the aero power (volumetric flow rate $\times$ pressure rise), the mechanical power (shaft torque $\times$ rotating speed), and the electric input power (current $\times$ voltage). These different power measurements help identify sources of inefficiency along the power train. 


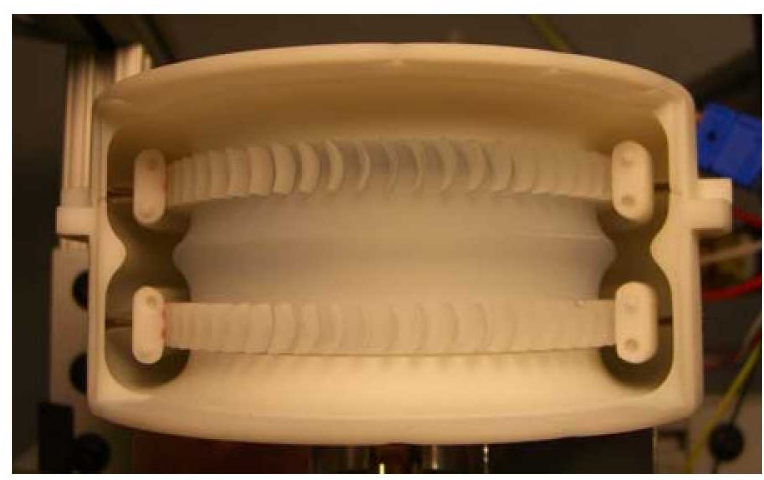

(a) Dual Impellers and Annual Flow Channels

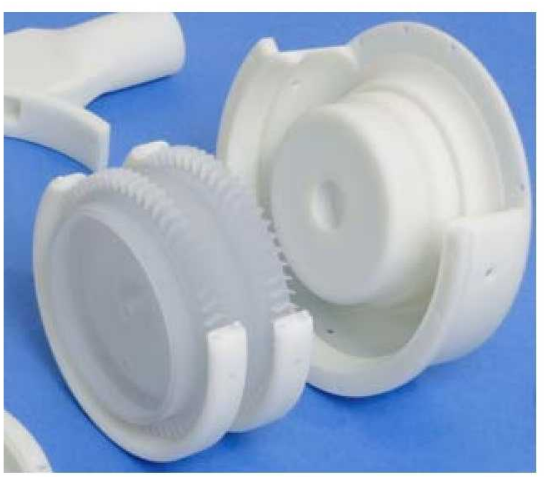

(b) Complete Assembly

Figure 7. Test Bed for Regenerative Blower Components.

Integral Blower Tests. We assembled a low-pressure flow loop that enabled testing of a complete regenerative blower under conditions that simulate operation in the ventilation subsystem of future EVA suits. We assembled prototype regenerative blowers and measured their head/flow performance and overall efficiency in this loop. Figure 9 shows the low-pressure flow loop. The facility includes a low-pressure-loss flow meter and a differential pressure transducer to measure the pressure rise produced by the blower. A rough vacuum pump was used to evacuate the loop to a sub-atmospheric pressure prior to the tests. For these tests, we could measure the overall electric power input but could not measure the mechanical power, since the blower was isolated inside the loop.

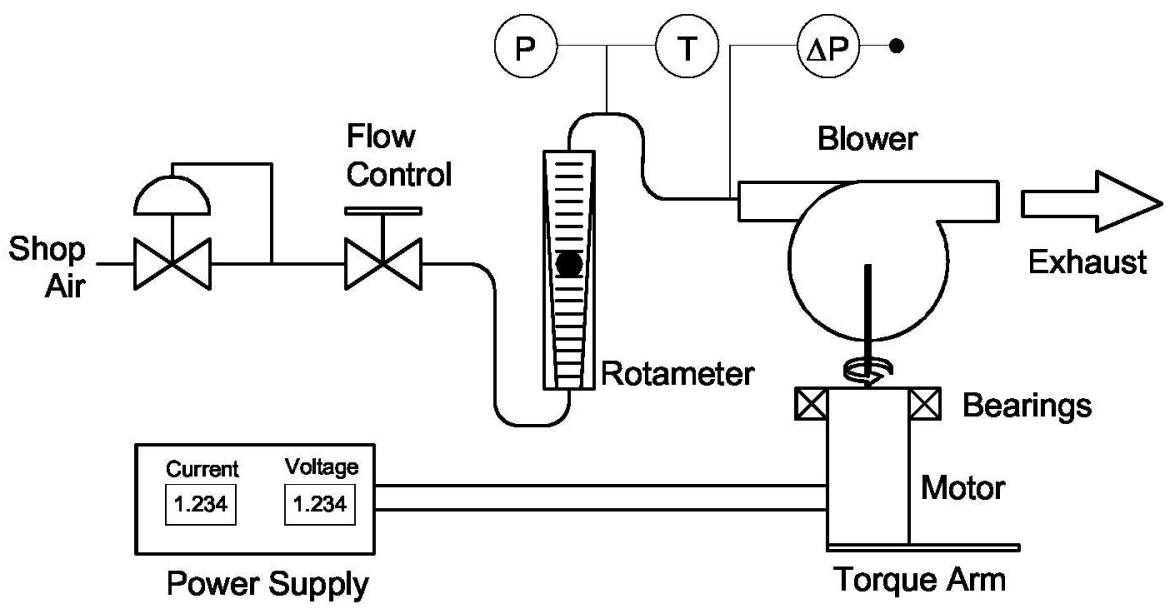

Figure 8. Test Setup for Separate Effects Tests. 


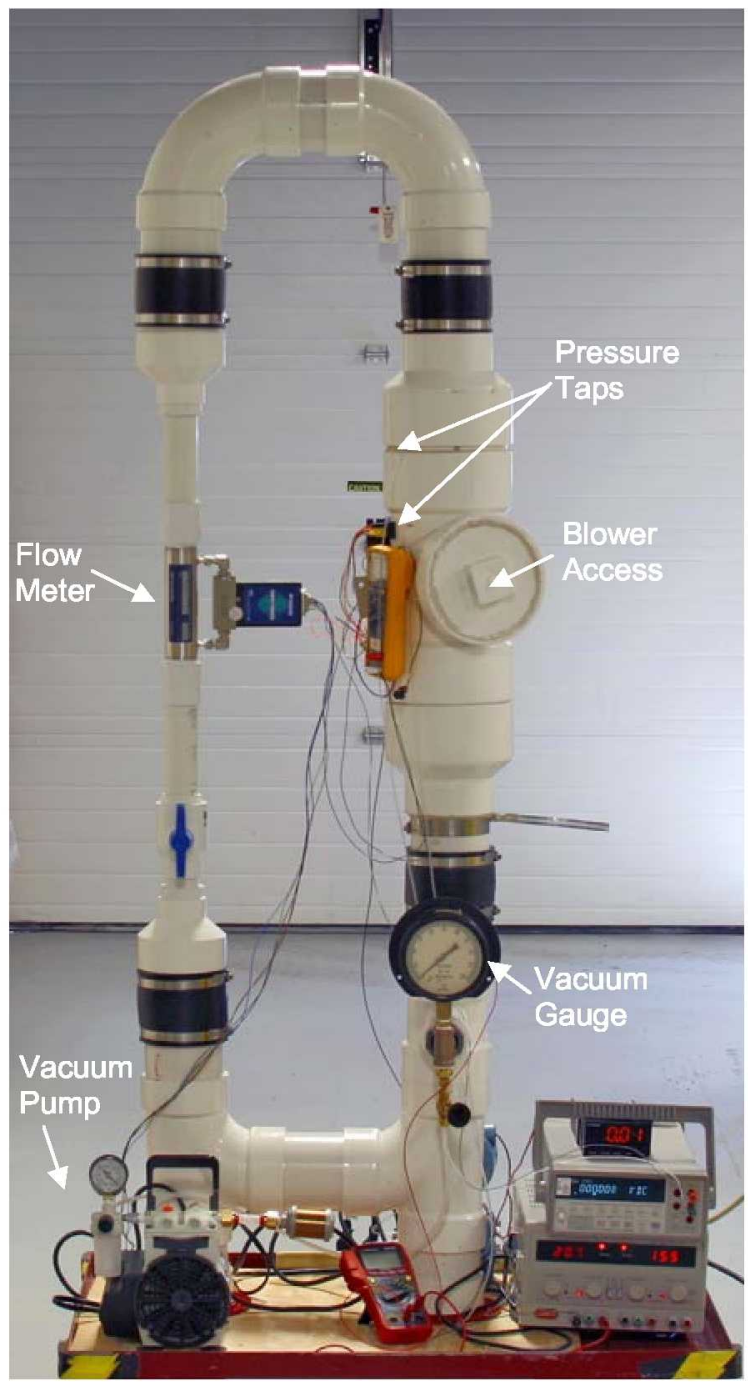

\section{Figure 9. Low-Pressure Loop for Blower Testing.}

\section{Test Results}

Test results show that it is feasible to achieve superior performance using a regenerative blower for the ventilation fan in future EVA suits. The final fan specifications are a good match for the capabilities of a compact regenerative blower operating at low speed. Separate effects tests produced data on impeller performance that we used to optimize the blade and blower design. Data from a proof-of-concept blower operating in prototypical conditions show that a compact regenerative blower can efficiently produce the flow rate and pressure rise needed for the ventilation subsystem. The data from tests of a typical inverter yield projections of the power conversion efficiency we can expect in the prototype blower. Along with the design and performance predictions for the direct current (DC) permanent magnet motor, these data enable us to project the expected efficiency of the prototype ventilation fan. Solid models for the complete blower assembly show the concept for a compact package and interface requirements.

\section{Separate Effects Test Results}

We used the separate effects test rig to investigate improvements to the impeller and flow channel design and to identify sources of inefficiency in the power train. Key results from these tests are:

- Wall friction in the flow channel is a major source of efficiency loss. Therefore, the flow channel shape should be toroidal/annular to minimize the ratio of wetted perimeter to flow area.

- Early impeller designs specified a blade spacing that could not effectively impart momentum to the gas. The ventilation fan should use blades with a smaller pitch. 
- Bearings consume a significant amount of power as well. The ventilation fan should incorporate precision ceramic bearings to minimize this power loss.

The first separate effects test investigated the importance of flow friction in the annular flow channel. The flow through this channel is complex and there are no published data that provide any guidance on optimizing the design

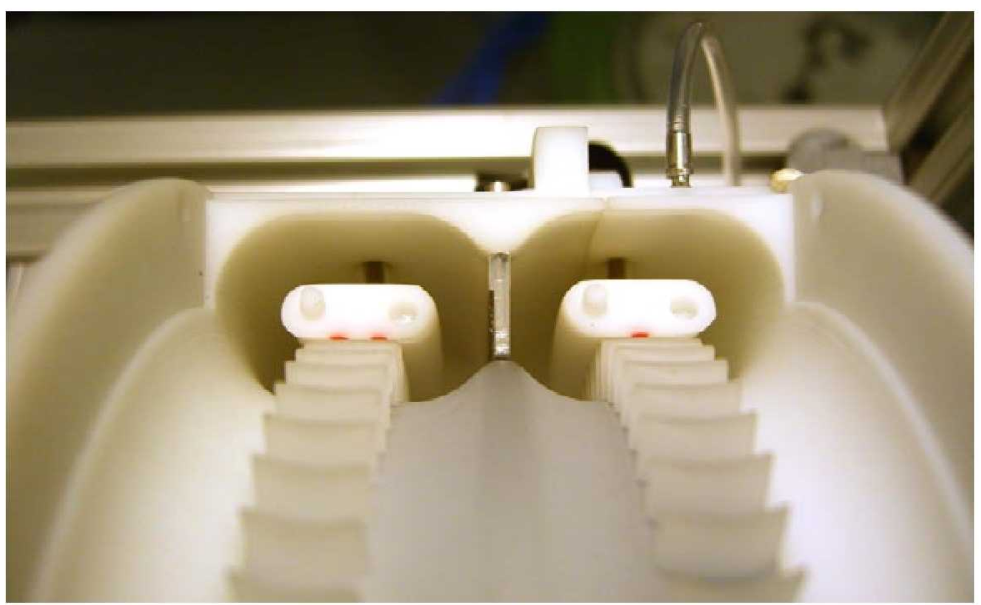

Figure 10. Flow Separator to Measure Effects of Wall Friction.

of the flow channel. To see the effects of flow friction, we built a flow separator that could be installed between the two parallel flow channels in the test bed (Fig. 10). Adding this component changed only the wetted perimeter of each flow channel without changing the overall flow pattern. Figure 11 shows the results of these tests. Adding the separator resulted in a $15-20 \%$ reduction in pressure rise at the same flow rate. Our conclusion is that wall friction is one of the most significant contributions to flow losses, and the most efficient blower will adopt a flow channel geometry with the minimum ratio of wetted perimeter to flow area. As a result, our proof-of-concept blower and the conceptual design for our prototype blower specify a circular, toroidal flow channel.

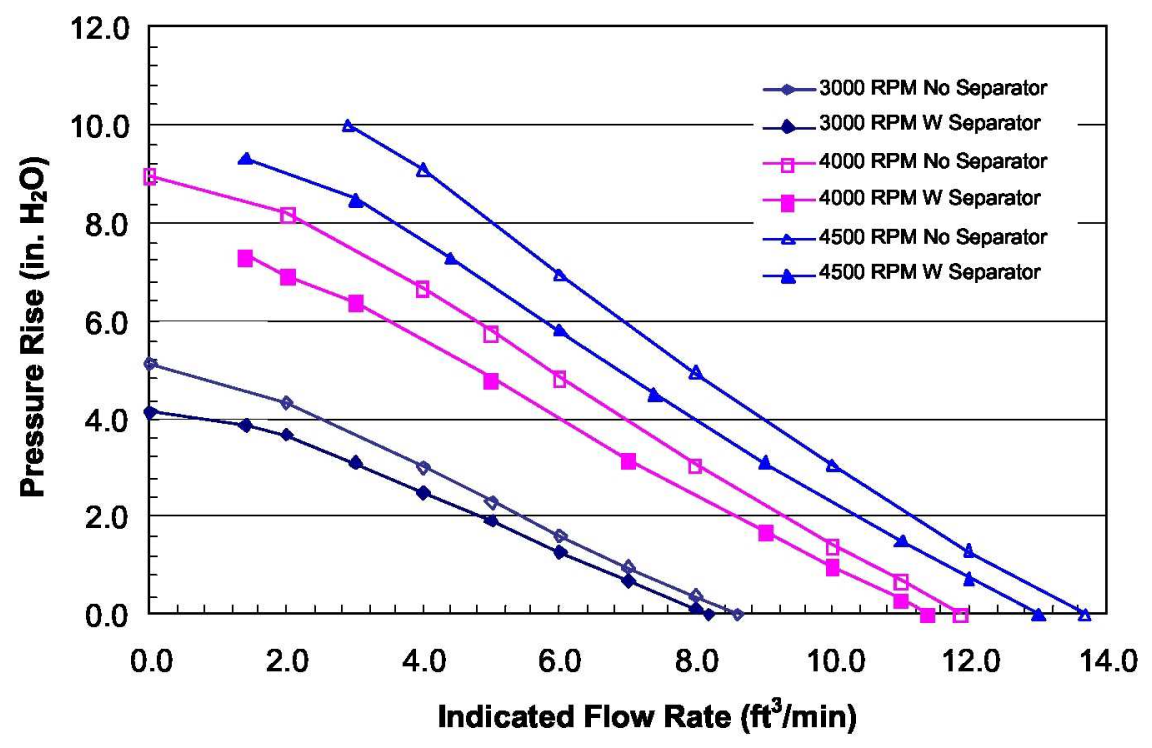

Figure 11. Effects of Flow Separator in Separate Effects Flow Rig.

The second set of separate effects tests investigated the effects of blade pitch on overall blower performance. We measured performance using two impellers that were identical except for the number of blades: 60 and 71 . Figure 12 shows the results of these tests. The blower with 71 blades produced significantly more pressure rise (25-30\%, 
depending on the flow rate) than the blower with 60 blades. We conclude from this test that the blade pitch in the 60 -blade impeller was too large to effectively change the gas velocity. To obtain detailed data on internal flow, we installed pressure taps at ten locations around the periphery of the blower. These taps allowed us to measure the static pressure in the annular flow channel, showing the local pressure gradient and the extent of effective pumping around the periphery. The pressure gradient is critical information for validation of our design models, and the effective pumping area indicates how well we have designed the gas inlet and outlet channels. Figure 13 shows the pressure distribution measured around the periphery of the blower when running with the 71-blade impeller. The data show a linear pressure rise around almost the entire periphery, with only a short developing region. Based on the results of these tests, our prototype blower design calls for 71 blades.

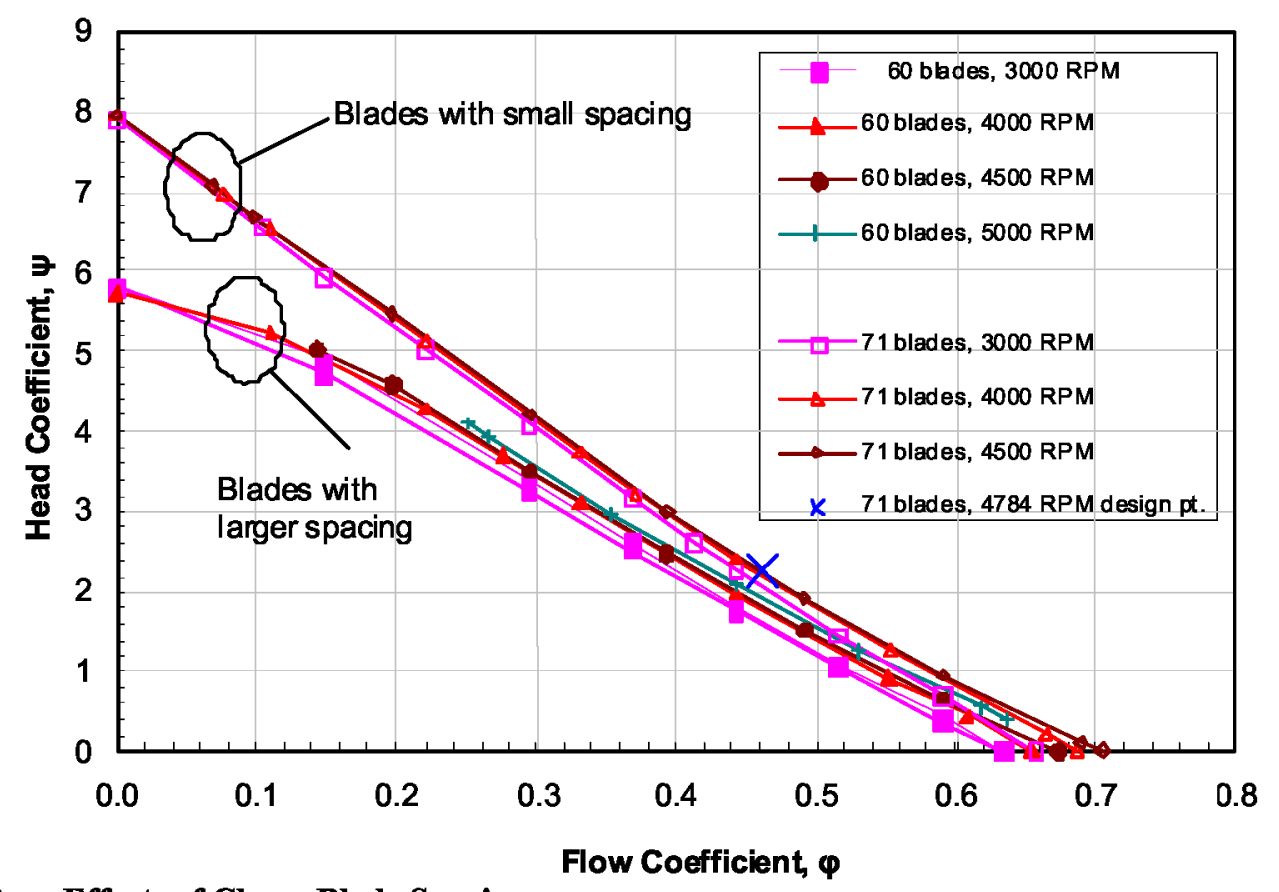

Figure 12. Effects of Closer Blade Spacing.

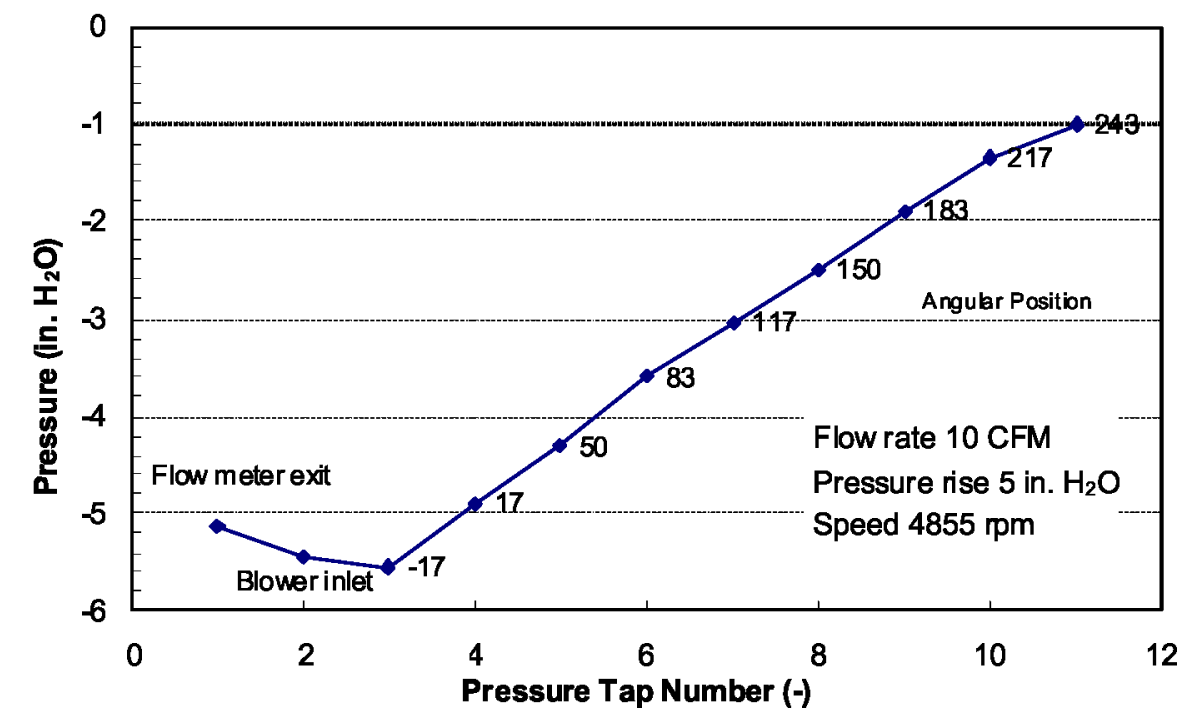

Figure 13. Pressure Distribution Around the 71-Blade Impeller.

American Institute of Aeronautics and Astronautics 


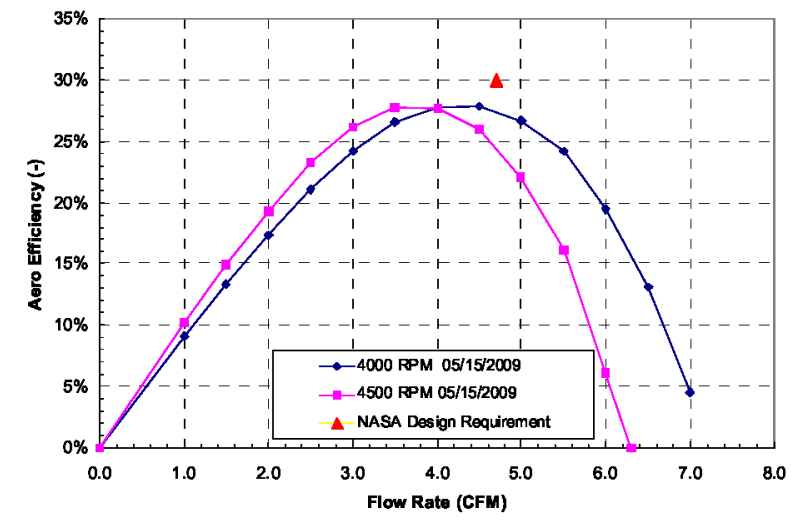

(a) With Bearings

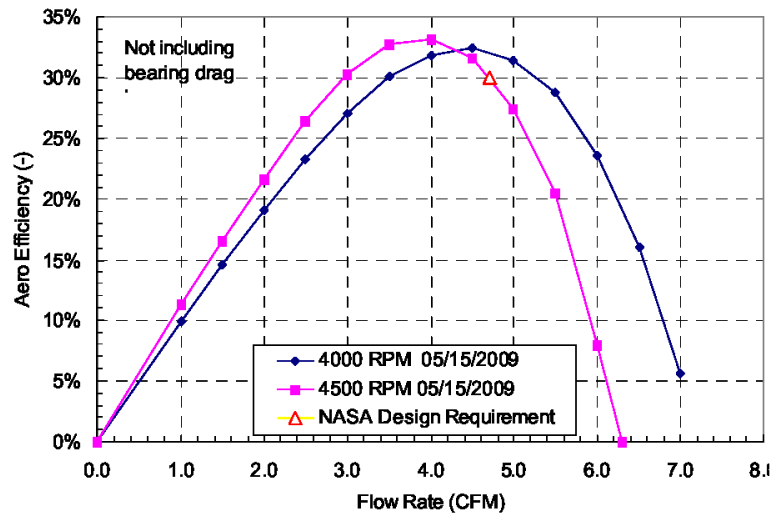

(b) Without Bearings

Figure 14. Overall Aerodynamic Efficiency of the Blower.

The final set of separate effects tests revealed that bearing and impeller disk drag losses were a major source of inefficiency. We measured the shaft power first without the impeller, then without the impeller and the bearings that support the impeller. The power consumed by the bearings and impeller disk is the difference between these two power measurements. We found that the losses are about $3 \mathrm{~W}$ at $4500 \mathrm{rpm}$. Figure 14 shows the results of these tests by plotting the measured aero efficiency of the blower at 4000 and $4500 \mathrm{rpm}$ (Fig. 14a) and then subtracting the bearing and impeller losses and re-plotting the aero efficiency (Fig. 14b). This calculation shows that the power consumed by the bearing and impeller drag alone is more than the power needed to be saved to achieve the efficiency goals for the ventilation fan.

\section{Blower Performance}

In the feasibility study described in this paper, performance testing focused on the current baseline performance requirements listed in Table 1. High efficiency while meeting the baseline requirements is key, since the blower will operate in this mode nearly all the time. Buddy mode operation is required only in rare emergencies, and will inevitably be inefficient for a blower that is optimized for a different operating point. Pressure drop and flow data from tests that include the baseline operating point can be easily scaled to predict the rotational speed required for buddy mode. Future tests of prototype blowers are planned that will confirm that the blower meets head/flow requirements and measure efficiency during buddy mode operation.

We assembled a proof-of-concept blower that incorporated an impeller with a single blade row (Fig. 15a) and incorporated the lessons learned from our separate effects tests. We installed the proof-of-concept blower in the lowpressure flow loop (Fig. 9, Fig. 15b) and measured its performance under prototypical, sub-atmospheric pressure using air to simulate the ventilation gas. Results show that the regenerative blower can achieve the performance needed for the ventilation subsystem at low rotating speed and low power consumption (only $9 \mathrm{~W}$ ). Performance at low pressure scales as expected to performance at atmospheric pressure.

Figure 16 shows that the blower performance measured in the low-pressure flow loop matches performance measured open-loop on the benchtop. The plot compares head/flow performance curves at three rotating speeds $(3180,4590$, and $5310 \mathrm{rpm})$ at atmospheric pressure. The data are nearly identical, giving high confidence in the head/flow data produced by the flow meter and pressure transducer in the low-pressure flow loop. 
Figure 17 plots head/flow performance data measured at five rotating speeds in the low-pressure flow loop at an absolute pressure of $4.3 \mathrm{psia}$. The head/flow requirements for the ventilation fan are also shown for comparison. These data show that the proof-of-concept blower can meet the current baseline requirements at a rotating speed of $5400 \mathrm{rpm}$ and the high flow rate requirements at a rotating speed of about $7000 \mathrm{rpm}$. Power consumed by the blower at $5310 \mathrm{rpm}$ while operating close to the ventilation fan design point was $9 \mathrm{~W}$. Since we expect additional improvements in flow performance as we develop the prototype fan, we believe that these rotating speeds are upper bounds for the prototype fan. Figure 18 plots the same data as Fig. 17 in nondimensional form, showing that the head/flow performance at different speeds can be predicted confidently using conventional scaling for the range of flow coefficients applicable to the ventilation fan. Based on these data, we expect that the blower can achieve the head and flow required for buddy-mode operation at a rotating speed of $9600 \mathrm{rpm}$.

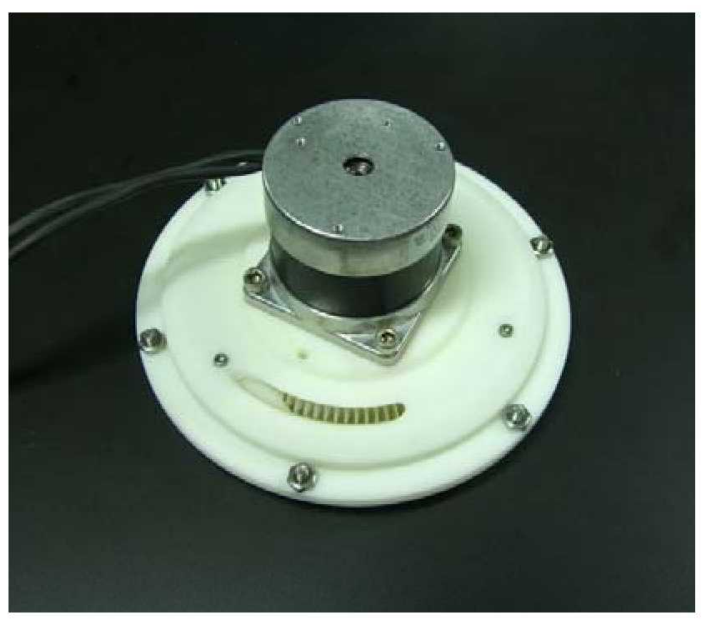

(a) Blower With Off-the-Shelf Motor and With Inlet and Exit Diffusers Removed

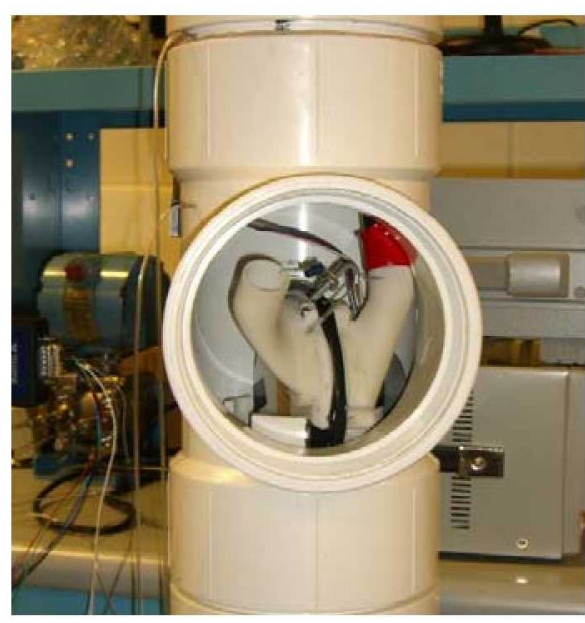

(b) Installed in Low-Pressure Flow Loop

Figure 15. Phase I Proof-of-Concept Regenerative Blower.

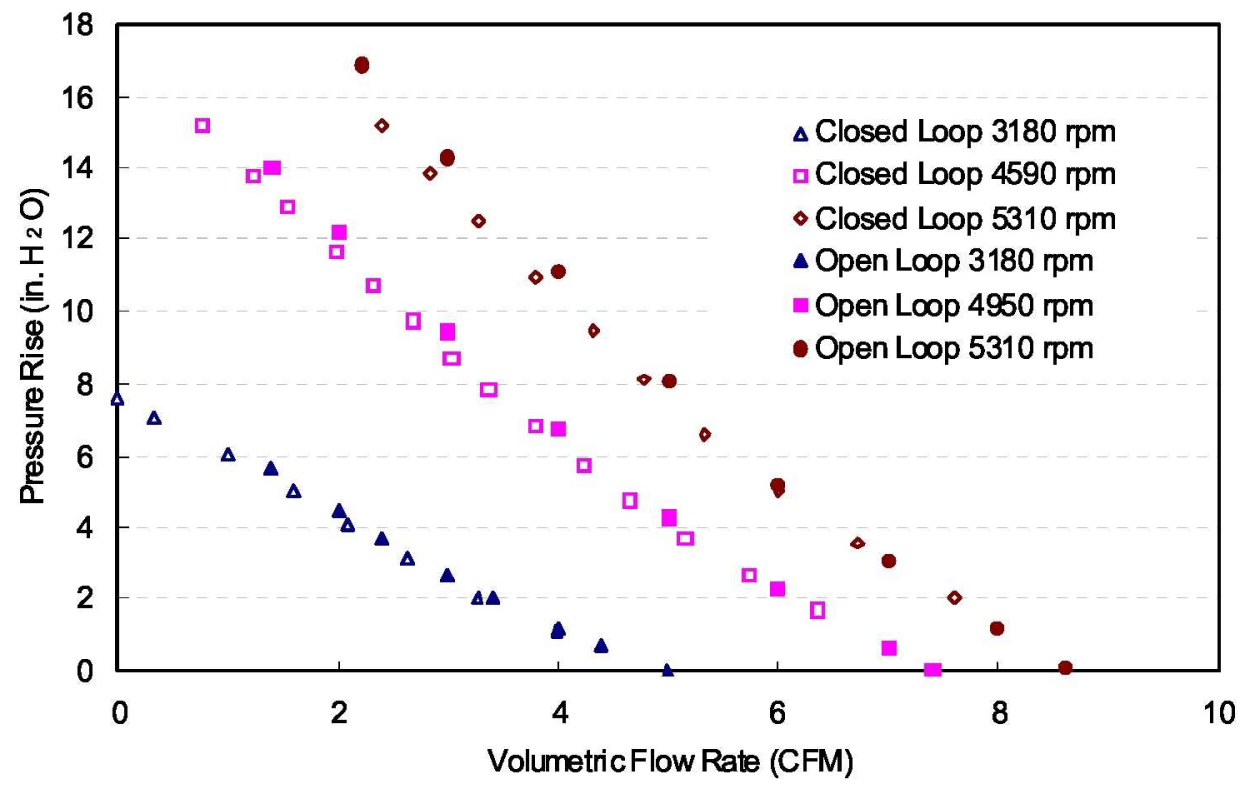

Figure 16. Open vs. Closed Low-Pressure Loop Performance Data. 


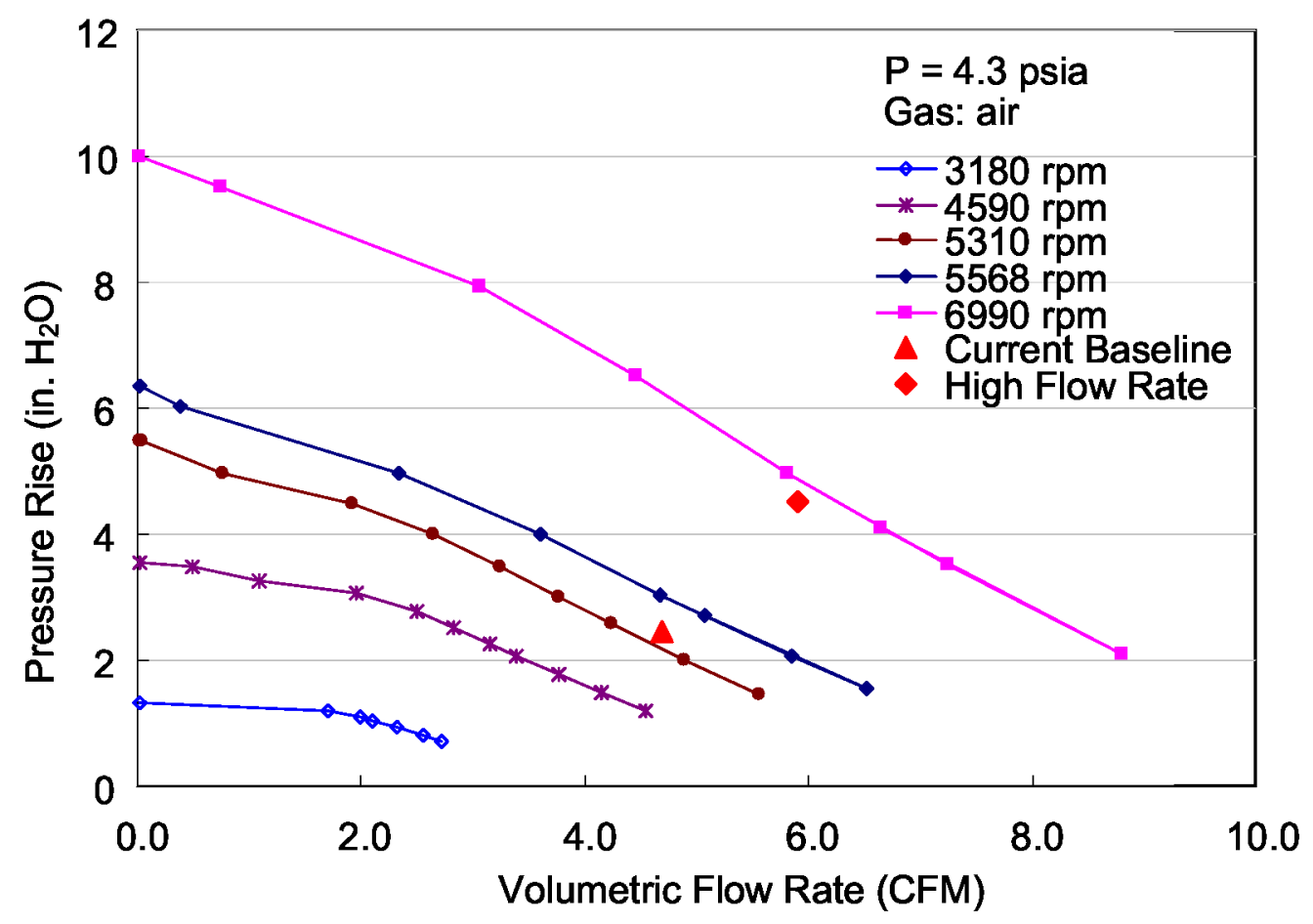

Figure 17. Low-Pressure Loop Performance Data vs. Design Requirements.

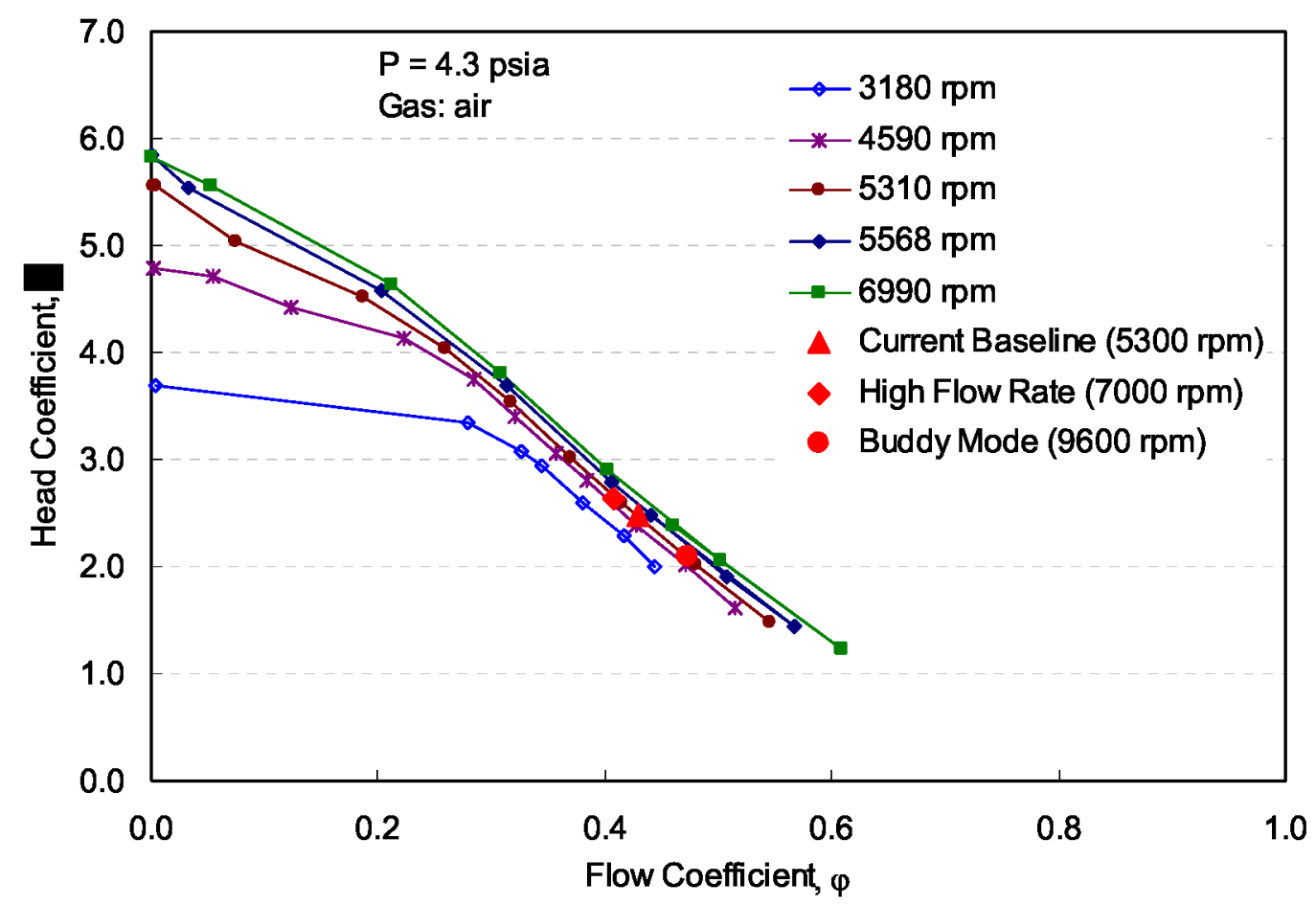

Figure 18. Low-Pressure Loop Performance Data in Nondimensional Format. 


\section{Prototype Blower Design}

Based on the proof-of-concept test data, we designed a prototype blower to meet the ventilation fan requirements. Table 2 lists the main design features, and Fig. 18 and Fig. 19 show several views of a solid model. The design goal is to provide optimal performance for the nominal flow conditions $\left(4.7 \mathrm{ft}^{3} / \mathrm{min}\right.$ and $2.7 \mathrm{in} . \mathrm{H}_{2} \mathrm{O}$ ) with the capability of short, infrequent operation under buddy mode when necessary. Normal rotating speed is $5400 \mathrm{rpm}$ with a power consumption of $6.9 \mathrm{~W}$. Our nominal design calls for a Torlon (poly(amide-imide)) impeller, but different materials (aluminum, graphite, machinable ceramics) can be substituted if needed to meet safety requirements.

\begin{tabular}{|c|c|c|}
\hline \multicolumn{3}{|l|}{ Performance } \\
\hline Operating mode & & Normal operation \\
\hline Flow rate & (actual $\mathrm{ft}^{3} / \mathrm{min}$ ) & 4.7 \\
\hline Pressure rise & (in. $\mathrm{H}_{2} \mathrm{O}$ ) & 2.7 \\
\hline Rotating speed & (rpm) & 5400 \\
\hline Power consumption & $(W)$ & 6.9 \\
\hline \multicolumn{3}{|l|}{ Design } \\
\hline Impeller material & & Torlon (poly(amide-imide)) \\
\hline Flow passage material & & Torlon (poly(amide-imide)) \\
\hline Impeller diameter at blade root & (in.) & 3.5 \\
\hline Bearings & & Silicon nitride hybrid bearing \\
\hline Bearing lubricant & & Krytox \\
\hline Pressure boost per stage & (in. $\mathrm{H}_{2} \mathrm{O}$ ) & 0.72 \\
\hline \multicolumn{3}{|l|}{ Mass Breakdown } \\
\hline Impeller & (g) & 60 \\
\hline Housing and flow passages & (g) & 185 \\
\hline Inlet/outlet assembly & (g) & 50 \\
\hline Bearings & (g) & 12 \\
\hline Motor and controller & (g) & 95 \\
\hline TOTAL & (g) & 402 \\
\hline Overall Volume & $(L)$ & 0.36 \\
\hline
\end{tabular}

Figure 18a shows a section view through the blower assembly, and Fig. 18b shows a view of the blower assembly with one of the cover shells removed. The section view shows how the impeller is mounted on a short shaft supported on both ends by radial ball bearings that are mounted in the cover shells. The annular flow channel is formed by features in both shells plus the toroidal flow channel core. The view with the cover shell removed shows the impeller blades and the annular flow channel. The inlet/exit/stripper seal assembly mounts around the toroidal core and lines up with the inlet and exit ports on the cover shells. 


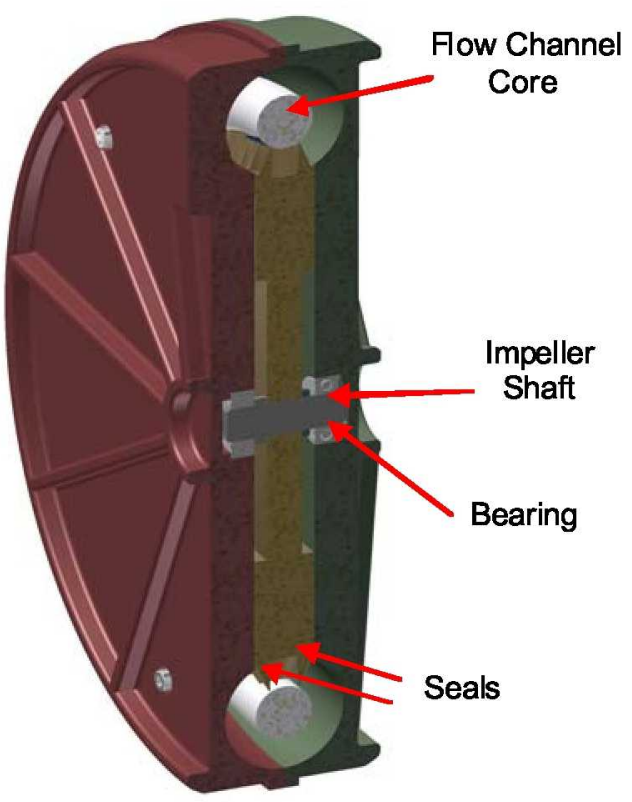

(a) Section View Through Blower Assembly

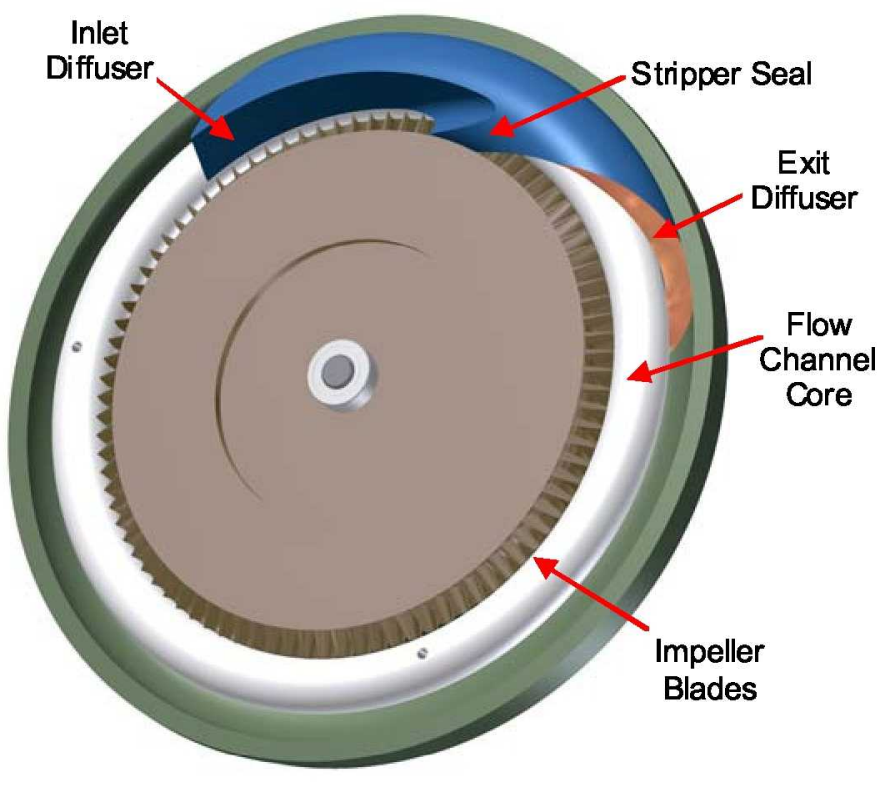

(b) View With Top Cover Removed

Figure 18. Design of the Prototype Blower (flat motor not shown).

The exploded view of the assembly (Fig. 19) shows how the parts fit together. Most of these components will probably be combined into subassemblies for ease of maintenance and repair. Because the tolerances for the regenerative blower are not extremely tight (adequate seals require clearances of only about $0.003 \mathrm{in} .(0.075 \mathrm{~mm})$ ), we expect that disassembly and reassembly will be simple and can be performed in situ if necessary for regular inspection, maintenance, or repair.

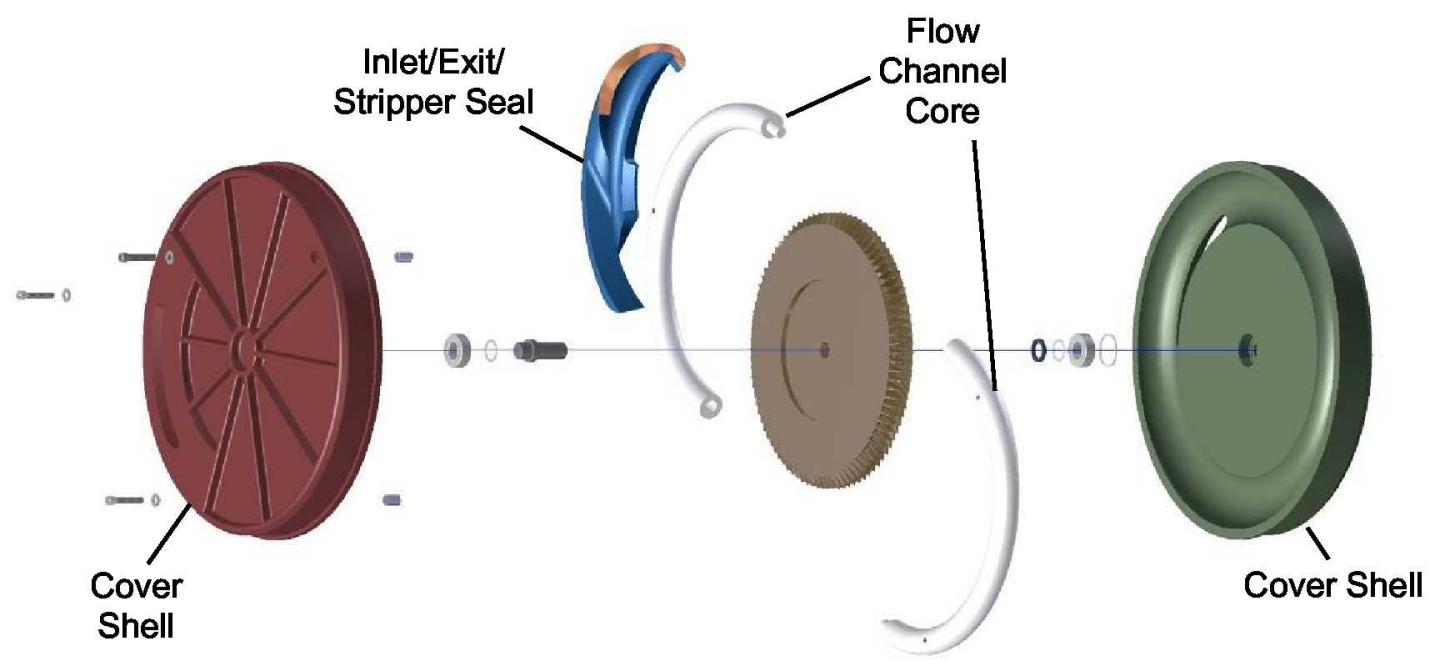

Figure 19. Prototype Blower—Exploded View (flat motor not shown). 
Motor Design. We designed a flat, permanent magnet motor that integrates with the blower to make a very compact package. The design is based on technology developed by Creare Inc. in a prior project to develop a very low-profile blower for electronics cooling. Scaling the motor design to the ventilation fan yields a very compact blower that will be simple to integrate with the PLSS.

Analysis of Efficiency and Power Consumption. The proof-of-concept blower has already demonstrated that the nominal head/flow requirements can be met with a power consumption of only $9 \mathrm{~W}$, corresponding to an overall efficiency of $16.5 \%$. Table 3 details our analysis of power consumption in the proof-of-concept blower based on measurements of the shaft power with and without the impeller and the measurements of inverter losses. We expect the main efficiency gains in the prototype blower will come from improvements in the bearings and the motor, since components in the proof-of-concept blower were off-the-shelf and far from optimum. Although we expect aero efficiency to improve in the prototype blower as well, Table 3 conservatively assumes no gain in aero efficiency. We believe that improved bearings and a more efficient flat motor design will be enough by themselves to meet the ventilation fan power goal of $8 \mathrm{~W}$ or less.

\begin{tabular}{|c|c|c|c|}
\hline \multicolumn{4}{|c|}{ Table 3. Analysis of Power Consumption } \\
\hline & & Proof-of-Concept & Projected Prototype \\
\hline \multicolumn{4}{|l|}{ Power Consumption } \\
\hline Aero power & $(\mathrm{W})$ & 1.5 & 1.5 \\
\hline Flow losses & (W) & 3 & 3 \\
\hline Bearing and impeller drag losses & (W) & 3 & 1.5 \\
\hline Motor losses & (W) & 1 & 0.4 \\
\hline Inverter losses & (W) & 0.5 & 0.5 \\
\hline TOTAL power input & (W) & 9 & 6.9 \\
\hline Aero efficiency & & 0.33 & 0.33 \\
\hline Mechanical efficiency & & 0.60 & 0.75 \\
\hline Motor efficiency & & 0.88 & 0.94 \\
\hline Inverter efficiency & & 0.94 & 0.94 \\
\hline Overall efficiency & & 0.17 & 0.22 \\
\hline
\end{tabular}

Significant reductions in bearing loss are feasible. Creare Inc. has developed a high-speed $(100,000 \mathrm{rpm})$ motor for a turbomolecular vacuum pump for NASA's Mars Science Laboratory mission. ${ }^{9}$ The combined losses in the bearings, motor, and inverter are less than $1.8 \mathrm{~W}$ at the design speed of $100,000 \mathrm{rpm}$ at atmospheric pressure. Since this motor uses the same precision ceramic bearings that we plan to use in the regenerative blower, this result shows that at least $1.2 \mathrm{~W}$ (the difference between the proof-of-concept bearings and the vacuum pump bearings) can be gained just by using the ceramic bearings.

Cooling. Aerodynamic losses are carried out of the blower by the ventilation gas and rejected to the thermal control subsystem via a heat exchanger (Figure 1). The motor and bearings will generate a small amount of heat due to electrical losses and friction. The current design calls for this heat to conduct through the blower housing to the peripheral flow channel, where it will also be absorbed by the ventilation gas and then rejected to the thermal control subsystem. This simple cooling approach, along with a peripheral seal that limits ventilation gas flow through the motor space, enhances safety by keeping dust out of the motor.

Oxygen Compatibility. Blower materials will be selected for compatibility with an oxygen environment. Because the blower operates at low speed and mechanical clearances are not very tight, the blower parts can be made from a wide range of materials. Oxygen compatibility and safety will be the major considerations in material selection. We have identified several candidate materials for the blower, which are listed in Table 4. Comprehensive testing and analysis will be needed prior to final selection, but we have made a preliminary selection of Torlon (poly(amideimide)) due to its good safety characteristics in an oxygen environment and mechanical stability. A thin anti-static layer (e.g., a sputtered gold film) can be applied if necessary. We have also made a preliminary selection of DuPont Krytox ${ }^{\circledR}$ lubricant for the bearings. Krytox is completely nonflammable, chemically inert, thermally stable, and completely safe to use in all areas of oxygen service. Krytox fluoropolymer lubricants have been used in oxygen service by NASA and the aerospace industry for over 40 years. 


\begin{tabular}{|c|c|c|}
\hline Component & Material & Justifications \\
\hline \multirow[t]{2}{*}{$\begin{array}{l}\text { Impeller and } \\
\text { housing }\end{array}$} & Anodized aluminum & $\begin{array}{l}\text { - } \quad \text { Aluminum oxide coating is relatively thick }(50 \mu \mathrm{m}) \text {. The coating is } \\
\text { extremely inert and wear resistant. } \\
\text { - } \quad \text { High thermal conductivity to dissipate heat } \\
\text { - } \quad \text { Excellent wear resistance } \\
\text { - } \quad \text { Extensively used in high pressure oxygen regulators }\end{array}$ \\
\hline & $\begin{array}{l}\text { Torlon (poly(amide- } \\
\text { imide)) with antistatic } \\
\text { coating }\end{array}$ & $\begin{array}{l}\text { - Lighter than aluminum and will not generate sparks upon impact of } \\
\text { high-speed particles } \\
\text { - High wear resistance, very high service temperature and high auto } \\
\text { ignition temperature } \\
\text { - } \quad \text { Polyimide is used as structural material for the space suit }\end{array}$ \\
\hline Bearing & Silicon nitride & $\begin{array}{l}\text { A very inert material. Used in the LOX turbo pump of the Space } \\
\text { Shuttle Main Engine } \\
\text { - Has a much longer service life than regular bearings }\end{array}$ \\
\hline Bearing lubricant & Krytox & $\begin{array}{l}\text { - Extensively used in oxygen service by NASA as pointed out in } \\
\text { DuPont's product literature } \\
\text { Extremely inert, nonflammable material with extremely low vapor } \\
\text { pressure }\end{array}$ \\
\hline $\begin{array}{l}\text { Motor windings } \\
\text { insulation }\end{array}$ & PTFE & $\begin{array}{l}\text { - Has excellent high temperature properties, is non-flammable and } \\
\text { is the most flexible of all insulations } \\
\text { Unlike Kapton, PTFE is not prone to wet-arc and dry-arc due to } \\
\text { abrasions and cuts }\end{array}$ \\
\hline $\begin{array}{l}\text { Inverter circuit } \\
\text { board coating }\end{array}$ & $\begin{array}{l}\text { Dow silicone } 3145 \\
\text { conformal coating }\end{array}$ & $\begin{array}{ll}\text { - } & \text { High service temperature } \\
\text { - } & \text { Flame retardant } \\
\text { - High dielectric strength }\end{array}$ \\
\hline
\end{tabular}

Safety Features. The regenerative blower will improve overall safety, due primarily to its low rotating speed and the materials that can be used due to the low rotating speed. Even at the rotating speed demonstrated in the Phase I contract $(5400 \mathrm{rpm})$, the tip speed of the impeller is only $28 \mathrm{~m} / \mathrm{s}$, which is less than the $30 \mathrm{~m} / \mathrm{s}$ limit, to avoid flammability concerns in an oxygen environment. We expect that efficiency improvements in Phase II will allow operation at even lower rotating speeds. The low rotating speed enables fabrication of the impeller from a wide variety of materials, limited primarily by fabrication methods. Therefore, the impeller can be made from stereolithography plastics or machined metals, graphite, or ceramics. Likewise, the materials for the remaining blower components (cover shells, stripper seal, and flow channel core) can be chosen primarily for good safety characteristics. The nominal bearing lubricant (Krytox) is also rated safe for operation in an oxygen environment.

\section{Assessment of Feasibility}

The results of this study show that it is quite feasible to develop a regenerative blower that will be an excellent match for the CSSS ventilation fan requirements. The proof-of-concept blower met the ventilation fan head/flow requirements at a rotating speed of $5400 \mathrm{rpm}$ and an electrical power input of $9 \mathrm{~W}$. This blower used a plastic housing and diffuser produced by stereolithography, common stainless steel ball bearings that generated significant drag, and an off-the-shelf motor and off-the-shelf inverter that were not optimized for low power. The key improvements needed to meet all the ventilation fan requirements are as follows:

- Reduce power consumption. The blower currently consumes about $1 \mathrm{~W}$ more power than the ventilation fan requirement. We believe that the planned switch from common stainless steel to precision ceramic ball bearings will easily reduce power by $1 \mathrm{~W}$ or more by reducing bearing drag. We expect additional improvements due to improved motor and inverter efficiency.

\footnotetext{
${ }^{5}<\mathrm{http}: / /$ www.nasa.gov/offices/oce/llis/0890.html>
} 
- Use materials that are oxygen-safe. The regenerative blower operates at low speed and can be constructed from a wide range of materials, with oxygen safety a primary design driver. We believe that oxygen-safe plastics such as Torlon with an anti-static coating are an excellent choice; however, oxygen-safe metals (e.g., Monel or anodized aluminum) or machinable ceramics (e.g., Macor) are also candidates. Stator windings will be coated with an oxygen-safe epoxy.

- Reduce size and mass. The prototype blower will use a custom, flat motor that minimizes overall size and mass of the blower assembly. The motor design is scaled up from Creare Inc.'s prior, successful demonstration of a flat motor for an electronics cooling fan. We expect this proprietary, custom motor to achieve significantly higher efficiency than the oversized, off-the-shelf motor demonstrated in our Phase I testing. The Phase II conceptual design based on this motor is significantly smaller and lighter than the ventilation fan requirements.

- Design for high reliability. Reliability of the blower will depend primarily on the bearings and seals. Our prototype design calls for ceramic bearings operating under low-load conditions with Krytox lubricant. The bearings themselves have a DN value of $2 \times 10^{6} \mathrm{~mm}-\mathrm{rpm}$ and therefore will easily run for 2500 hours at $5400 \mathrm{rpm}$ with extremely low probability of failure. ${ }^{6}$ Creare Inc. has proven extremely long life of this ceramic bearing in a $100,000 \mathrm{rpm}$ turbomolecular pump, demonstrating a service life longer than 4000 hours. Krytox is chemically inert and will not degrade by reaction with oxygen even at high temperature $\left(900^{\circ} \mathrm{F}\right)$.

- Demonstrate performance, reliability, and safety. As part of the prototype development effort that began in January 2010, we plan to demonstrate that the regenerative blower meets the ventilation fan head/flow requirements while meeting the size, mass, and power consumption limits. We will operate the blower in an environment with simulated lunar dust and show that the blower performance does not degrade. We will life-test prototypical bearings in a prototypical environment and show that there is no degradation for long run-times. We will operate the blower in a pure oxygen environment and show that the materials are compatible with oxygen.

\section{Conclusions}

Regenerative blowers offer significant technical benefits and have the potential to improve performance of the ventilation subsystem in future EVA suits. This effort has shown that the regenerative blower approach is very feasible:

- Regenerative blowers are the optimal design choice for ventilation fans in future EVA suits. This conclusion is based on an analysis of the specific speed and diameter derived from the design requirements and comparison with general design maps for turbomachines.

- A regenerative blower can meet performance requirements for the ventilation subsystem in the Constellation Space Suit while meeting key design goals for small size, low mass, low speed, ruggedness and reliability, and good safety characteristics for operation in an oxygen environment.

- We proved feasibility of this approach through separate effects tests, fabrication method development, demonstration tests of complete blowers under prototypical conditions, and blower design.

In our ongoing program to produce a prototype blower, we plan to optimize the design of the components and the overall blower, produce performance data, demonstrate safe operation in an oxygen environment, and deliver a prototype to NASA for further testing and evaluation by the end of 2011.

\section{Acknowledgments}

The work described in this paper was sponsored by the Space Suit and Crew Survival Systems Branch at the NASA Lyndon B. Johnson Space Center and the U.S. Army Natick RD\&E Center.

\section{VIX. References}

\footnotetext{
${ }^{1}$ Cooke, D., "Constellation Space Suit System Contract Award Announcement," NASA Exploration Systems Mission Directorate, <www.nasa.gov>, June 12, 2008.
}

\footnotetext{
${ }^{6} \mathrm{DN}$ is the product of inner race diameter (D) and rotating speed $(\mathrm{N})$. The DN value for a bearing does not have quantitative significance for reliability, but the number is given by manufacturers because it is useful for a rule of thumb assessment of a bearing's suitability. In the case of the ventilation fan, the ceramic bearings have $\mathrm{DN}=2 \times 10^{6} \mathrm{~mm}$-rpm, so a $12.5 \mathrm{~mm}$ bearing could operate with good reliability at speeds up to $160,000 \mathrm{rpm}$, about 30 times greater than the planned operating speed.
} 
${ }^{2}$ Bailey, P., "Constellation Space Suit Element Portable Life Support System (PLSS) Schematic Selection Study," NASA JSC-65443, CTSD-CX-0005, February 2007.

${ }^{3}$ Barnes, B., Conger, B., Leavitt, G., Autrey, D. and Wells, J., "Constellation Space Suit Element PLSS Baseline Schematics and Internal Interfaces," Rev. B, CTSD-CX-5117, JSC-65563, NASA/Johnson Space Center, September 2009.

${ }^{4}$ Kempf, R., Vogel, M. and Paul, H., "Ventilation Transport Trade Study for Future Space Suit Life Support Systems," 38 International Conference on Life Support Systems, SAE International, Paper No. 2008-01-2115, June 29-July 2, 2008.

${ }^{5}$ Paul, H., Jennings, M. and Vogel, M., "Fan Performance Testing and Oxygen Compatibility Assessment Results for Future Space Suit Life Support Systems," 2009 International Conference on Environmental Systems, SAE International, Paper No. 2009-01-2448, July 2009.

${ }^{6}$ Balje, O. E., Turbomachines: A Guide to Design, Selection, and Theory, John Wiley \& Sons, Inc.: New York, 1981.

7Sixsmith, H. and Altmann, H., "A Regenerative Compressor," Vol. 99, No. 3, J Eng Ind., ASME Trans., 1977 , pp. 637-647.

${ }^{8}$ Swift, W. L., Nutt, W. E. and Sixsmith, H., "A Helium Regenerative Compressor," Advances in Cryogenic Engineering, (Albuquerque, NM), edited by P. Kittel, Vol. 39A, Plenum Press, New York, NY, 1994, pp. 901-908.

${ }^{9}$ Kline-Schoder, R. J. and Sorensen, P. H., "Miniature High Vacuum Pumps for Analytical Instruments," Presented at: 6th Harsh-Environment Mass Spectrometry Workshop, Coco Beach, FL, 17-20 Sep 2007.

\section{Contact}

Dr. Michael G. Izenson is a Principal Engineer at Creare Inc., where he has developed innovative thermal technologies for over 20 years. E-mail address: mgi@creare.com.

\section{Acronyms}

CFD: Computational Fluid Dynamics

CFM: $\quad \mathrm{ft}^{3} / \mathrm{min}$

CSSE: Constellation Space Suit Element

CSSS: Constellation Space Suit System

DN: $\quad$ Bearing diameter $\times$ rotating speed

EVA: $\quad$ Extravehicular Activity

LCVG: Liquid Cooling and Ventilation Garment

PLSS: Portable Life Support System

PTFE: Polytetrafluoroethylene (Teflon)

RPM: revolutions/min 